\title{
AN AUTOZEROING AMPLIFIER USING PFET HOT-ELECTRON INJECTION
}

\author{
Paul Hasler, Bradley A. Minch, Chris Diorio, and Carver Mead ${ }^{1}$ \\ ${ }^{1}$ California Institute of Technology \\ Pasadena, CA 91125 \\ paul@hobiecat.pcmp.caltech.edu
}

\begin{abstract}
We have developed an amplifier which removes its "offset" as a natural part of its operation by modifying the charge on a floating gate. The charge on the floating gate is adapted by a combination of electron tunneling and hot-electron injection, resulting in a nonlinear high-pass filter with a cutoff frequency less than $1 \mathrm{~Hz}$. We show experimental data from this autozeroing amplifier for various input waveforms, and an analytical model which fits the output waveforms. This autozeroing amplifier is a single-input case of a continuous learning circuit.
\end{abstract}

\section{INTRODUCTION}

We have developed an amplifier which removes its "offsets" as a natural part of its operation by modifying the charge on a floating gate. Offsets, including drifts due to temperature, are often a difficult problem in designing analog circuits, and it is desirable if not essential to eliminate them. Previous autozeroing techniques have relied on clocking schemes which periodically compute the offset and then subtract it from the input [1].

The approach presented here has several important applications. This autozeroing amplifier is the first known application of hot-electron injection in pFETs. Until now, pFET hot-electron injection has only been considered as a source of MOSFET degradation [2]. This amplifier is a singleinput case of a family of continuous learning circuit. The ability to naturally equalize the charge on a floating gate to produce a desired source current makes floating gate MOS circuits, as discussed in [3], more practical. This autozeroing amplifier does not require continuous clocking schemes, which reduces the noise levels.

\section{OVERVIEW}

Figure 1 shows the autozeroing amplifier. The open loop amplifier consists of a pFET input transistor and an nFET used as a current source. With capacitive feedback, the input signal is amplified by a closed loop gain approximately equal to $-\frac{C_{1}}{C_{2}}$. The maximum gain of the amplifier is limited by the open loop gain and the parasitic $C_{2}$ due to the floating gate overlap of the drain region. The steadystate output voltage is kept nearly constant by changing the charge on the floating gate; hence, the circuit behaves as a nonlinear high-pass filter with a very long time constant. The complimentary combination of tunneling and injection processes adds or removes electrons from the floating gate as needed at various 'slow' rates.

Two conditions must be satisfied for the circuit to be in equilibrium. First, the current the pFET must equal the current in the nFET. We define this quiescent source current as $I_{s 0}$. Second, the injection current must equal the tunneling current. We define $I_{i n j 0}$ as the quiescent injection current which must equal $I_{t w n}$, the quiescent tunneling current, at equilibrium. Since the tunneling and injection currents are much smaller than the source current of the FETs and are charging similar capacitances, the first condition is satisfied much faster than the second condition. The frequency range over which the first condition is satisfied but the second condition is not satisfied is defined to be the operating region of the circuit. The combination of electron tunneling and pFET hot-electron injection applies the appropriate negative feedback to stablize the output voltage such that the second condition is satisfied.

The steady state output voltage and the cutoff frequency are explicitly set by $V_{t u n}$ and the power supply voltage. Increasing $V_{t u n}$ increases the tunneling current, which decreases the settling time, but also drops the steady-state voltage since the pFET must have more drain voltage for the injection current to equal the tunneling current. Increasing the power supply voltage increases the steady-state drain to source voltage, thereby increasing the injection current. The larger power supply voltage decreases the tunneling current by decreasing the voltage accross the tunneling capacitor, which would increase the settling time and increase the steady state voltage. We present data for the autozeroing amplifier from a $2 \mu m n$-well CMOS process from Orbit. Typical operating values for $V_{t u n}$ were between $33 V$ to $40 \mathrm{~V}$ and for $V_{d d}$ were $6 \mathrm{~V}$ to $12 \mathrm{~V}$. For processes with smaller line widths the typical operating voltages will decrease due to the thinner gate oxide and higher substrate doping.

\section{CIRCUIT MODEL OF PFET HOT-ELEC'TRON INJECTION AND ELECTRON TUNNELING}

Before considering the behavior of the autozeroing amplifier, we will review the processes of electron tunneling and hot-electron injection in pFETs. First, we consider electron tunneling. Increasing the voltage on $V_{t u n}$ reduces the effective barrier width across the oxide, which increases the probability of the electron tunneling through the barrier. Typical values for $V_{t u n}$ are $33 \mathrm{~V}$ to $40 \mathrm{~V}$ for a $44 \mathrm{~nm}$ oxide. As in [4], we approximate the tunneling current for a fixed $V_{t u n}$ by

$$
I_{t u n}=I_{t u n 0} e^{\frac{\Delta v_{f g}}{V_{x}}}
$$

where $V_{x}$ is a tunneling device parameter, and $\Delta V_{f g}$ is the change in the floating gate voltage from the quiescent floating gate voltage. A typical value of $V_{x}$ is $1 V$ for our operating conditions. 


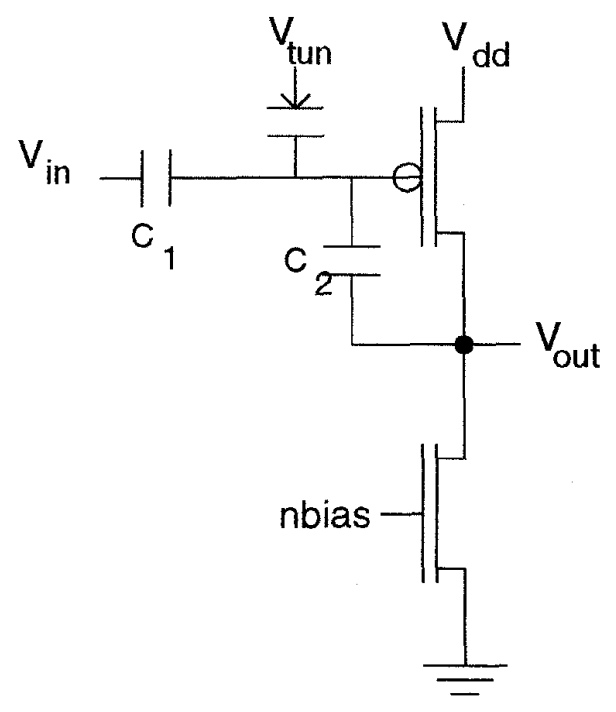

Figure 1. Circuit of the autozeroing amplifier using the hot-electron injecting pFET. The ratio of $C_{2}$ to $C_{1}$ sets the gain of this inverting amplifer. The nFET is a current source and sets the current through the pFET. Steady state occurs when the injection current equals the tunneling current.

Next we consider the modeling of pFET hot-electron injection. Hot-hole impact ionization creates electrons at the drain edge of the depletion region. These secondary electrons travel back into the channel region gaining energy as they go. When their energy exceeds that of the $\mathrm{SiO}_{2}$ barrier, they can be injected through the oxide to the floating gate. The hole impact ionization current is proportional to the source current, and is an exponential function of the voltage drop from channel to drain. We express this relationship as

$$
I_{\text {impact }}=I_{s} e^{f_{1}\left(\Phi_{d c}\right)} .
$$

The injection current is proportional to the hole impact ionization current and is an exponential function of the voltage drop from channel to drain. We express this relationship as

$$
I_{i n j}=I_{i m p a c t} e^{f_{2}\left(\Phi_{d c}\right)} .
$$

The injection current is a weak function of the floating gate voltage for a given source current and $\Phi_{d c}$, so we will neglect the dependance for this application.

Figure 2 shows measured injection efficiency for several source currents. Injection efficiency is the ratio of the injection current to source current. The functions $f_{1}, f_{2}$ are approximately linear over a $1--2 V$ swing in $\Phi_{d c}$. With this linear approximation we write the hot-electron injection current as

$$
I_{i n j}=I_{i n j 0} \frac{I_{s}}{I_{s 0}} e^{\frac{-\Delta \Phi_{d c}}{V_{i n j}}}
$$

where $V_{i n j}$ is a measurable injection device parameter, and $\Delta \Phi_{d c}$ is the change in $\boldsymbol{\Phi}_{d c}$ from the bias level. A typical value for $V_{i n j}$ is $250 \mathrm{mV}$. An increasing input signal will increase the pFET surface potential by capacitive coupling. Increasing the pFET surface potential will increase

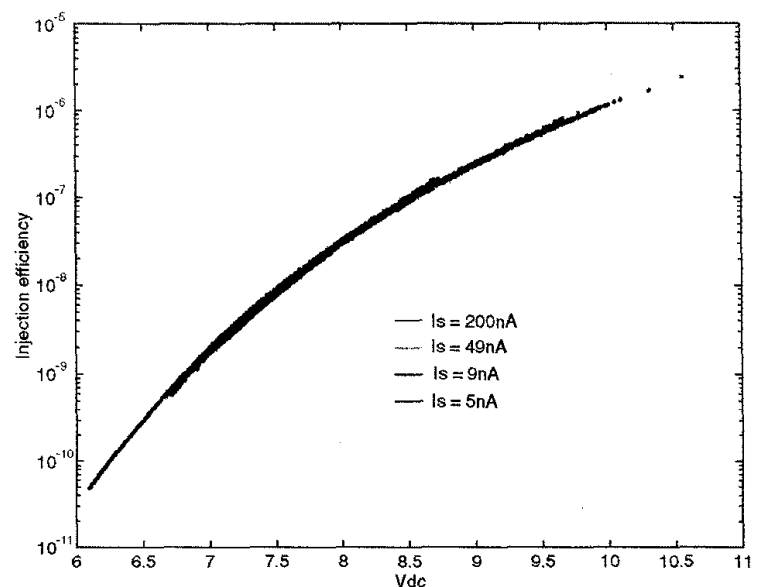

Figure 2. Measured data of pFET injection efficiency verses the drain to channel voltage for four source currents. Injection efficiency is the ratio of injection current to source current. At $\Phi_{d c}$ equal to $8.2 \mathrm{~V}$, the injection efficiency increases a factor of $e$ for an increase $\Phi_{d c}$ of $250 \mathrm{mV}$.

the source current thereby decreasing $\Phi_{d c}$ for a fixed output voltage and lowering the injection efficiency. As in [4], we model the injection current as the $\alpha$ power of the source current.

$$
I_{i n j}=I_{i n j 0}\left(\frac{I_{s}}{I_{s 0}}\right)^{\alpha} e^{\frac{-\Delta V_{d}}{V_{i n j}}}
$$

where $I_{s 0}$ is the quiesent source current, $V_{d}$ is the drain voltage, and $\alpha$ is $1-\frac{U_{2}}{V_{i n j}}$. A typical value of $\alpha$ is 0.9 , which is consistant with $V_{i n j}$ equal to $250 \mathrm{mV}$.

\section{BEHAVIOR OF THE AUTOZERO AMPLIFIER}

To model the autozero amplifier, we make two important simplifications. First, the open-loop gain from the floating gate to the output is can be large; a typical open loop gain is 300 . To keep the output voltage between the supply rails the floating gate must have small voltage swings; for typical open loop gains, the floating-gate voltage is confined to a $30 \mathrm{mV}$ swing. Thus we will approximate the floating-gate voltage as a constant. Second, the floating-gate voltage being nearly fixed implies the source current varies only slightly. The quiesent source current $\left(I_{s 0}\right)$ is set by the nFET current source. We model the injection current as having a source current set at $I_{s 0}$, which from (1) and (5) results in the floating gate current

$$
I_{t u n}-I_{i n j}=I_{t u n 0}\left(1-e^{-\frac{\Delta V_{a u t}}{V_{i n j}}}\right)
$$

where $I_{t u n 0}=I_{i n j 0}$ for the circuit to be in equilibrium.

By setting the current into the floating gate equal to the current leaving the floating gate, we can model the output voltage of the amplifier, $V_{o u t}$, in terms of $V_{i n}$. The total floating-gate current is the sum of the capacitive coupling 


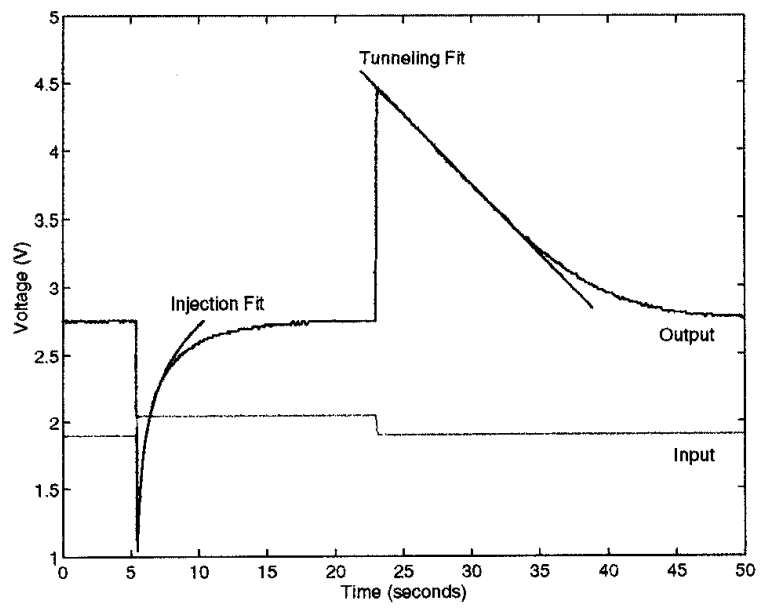

Figure 3. Response of autozero amplifiers to an upgoing and downgoing step function. The adaptation in response to an upward step results from electron tunneling; the adaptation in response to a downward step results from pFET hot-electron injection. This amplifier had a gain of 11.2 , as seen by comparing the ratio of the output and input steps. We plot the curve fits of the simplified expressions of (12) where either tunneling or injection dominates the restoration process. From the fits, we get $\tau$ as $4.3 \mathrm{~s}$ and $I_{\text {tun } 0}$ as $50 \mathrm{fA}$. The value of $\tau$ can be set as slow as many days.

currents of the input and output terminals plus the tunneling and injection currents. So, we write

$$
C_{1} \frac{d V_{\text {in }}}{d t}+C_{2} \frac{d V_{o u t}}{d t}=-I_{t u n 0}\left(1-e^{-\frac{\Delta V_{\text {out }}}{V_{\text {inj }}}}\right)
$$

To solve ( 7) we make the following change of variables

$$
X=e^{\frac{\Delta V_{\text {out }}}{V_{i n j}}}
$$

The resulting equation for $X$ is a linear, first-order differential equation with variable coefficients

$$
\tau \frac{d X}{d t}+\frac{\tau A_{v} X}{V_{i n j}} \frac{d V_{i n}}{d t}=1-X
$$

where $\tau$ is $\frac{C_{2} V_{i n j}}{I_{t u n 0}}$, and $A_{v}$ is the closed-loop gain of the amplifier, which is $-\frac{C_{1}}{C_{2}}$. In the next two sections, we will show the amplifier response to various inputs.

\section{RESPONSE TO A VOLTAGE STEP}

Consider the output response to a voltage step in the input. Assume the output voltage has initially adapted to its steady-state value $\left(\Delta V_{\text {out }}=0\right)$. Solving with this initial condition is equivalent to starting the output voltage proportional to the input voltage step times the amplifier gain and tracking the relaxation of the output voltage to the steady-state value. We denote this effective initial condition in the output voltage by $V_{\text {out }}\left(0^{+}\right)$. We denote the corresponding initial condition in $X$ by

$$
X\left(0^{+}\right)=e^{\frac{V_{\text {out }}\left(0^{+}\right)}{V_{\text {inj }}}},
$$

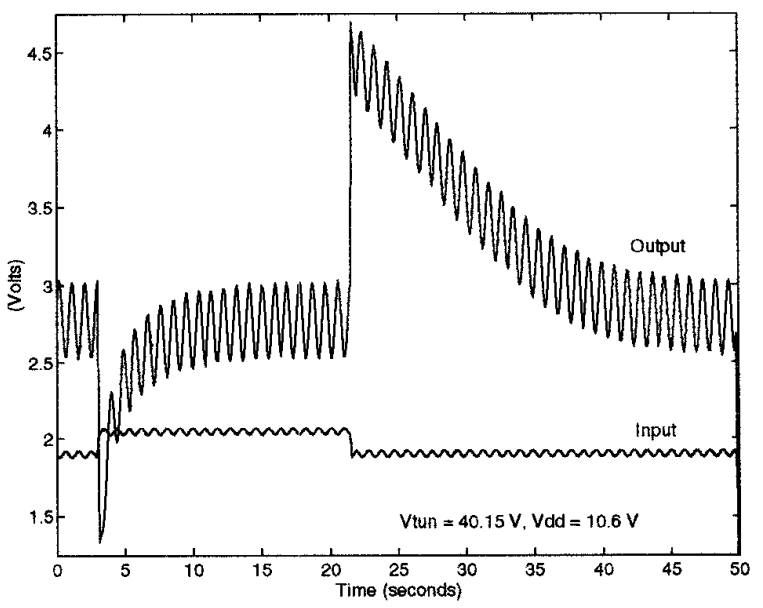

Figure 4. Response of the autozero amplifer to a $1 \mathrm{~Hz}$ sinewave superimposed on an upgoing voltage pulse. This autozero voltage amplifier had a closedloop gain of 11.2 .

For a downward input step, $X\left(0^{+}\right)$is greater than one, and for an upward input step, $X\left(0^{+}\right)$is less than one. We write the resulting differential equation as

$$
\left\{\begin{array}{l}
\tau \frac{d X}{d t}=1-X \\
X(0)=X\left(0^{+}\right)
\end{array}\right.
$$

The solution to this equation in terms of $\Delta V_{\text {out }}$ is

$$
\Delta V_{o u t}(t)=V_{i n j} \operatorname{Ln}\left(1+\left(X\left(0^{+}\right)-1\right) e^{-\frac{t}{\tau}}\right)
$$

where $\Delta V_{\text {out }} \rightarrow 0$ as $t \rightarrow \infty$.

The circuit has three interesting regions of operation which are approximated by

$$
\Delta V_{\text {out }} \approx\left\{\begin{array}{cl}
\Delta V_{\text {out }}\left(0^{+}\right) e^{-\frac{t}{\tau}} & X\left(0^{+}\right) \approx 1 \\
\Delta V_{\text {out }}\left(0^{+}\right)-\frac{I_{\text {tun }} t}{C_{2}} t & X\left(0^{+}\right) \gg 1 \\
V_{\text {inj }} L n\left(X\left(0^{+}\right)+\frac{t}{\tau}\right) & X\left(0^{+}\right) \ll 1
\end{array} .\right.
$$

The first case is when the tunneling nearly equals the injection current. The solution in this region is a familier exponential decay of a linear system. The second case is when the tunneling current dominates the injection current. The solution in this region is due to the constant tunneling current removing charge from the floating gate. The third case is when the injection current dominates the tunneling current. Figure 2 shows a measured response to an input pulse with curve fits to the regions where either the tunneling or injection current dominates.

\section{FREQUENCY DEPENDANT RESPONSE OF THE AUTOZERO AMPLIFIER}

If the input changes on a timescale that is much faster than the adaption due to the tunneling and injection processes, then the output is an amplified version of the input signal. Figure 4 shows the response to a $1 \mathrm{~Hz}$ sine wave superimposed on an input pulse. The amplifier adapts to the pulse 


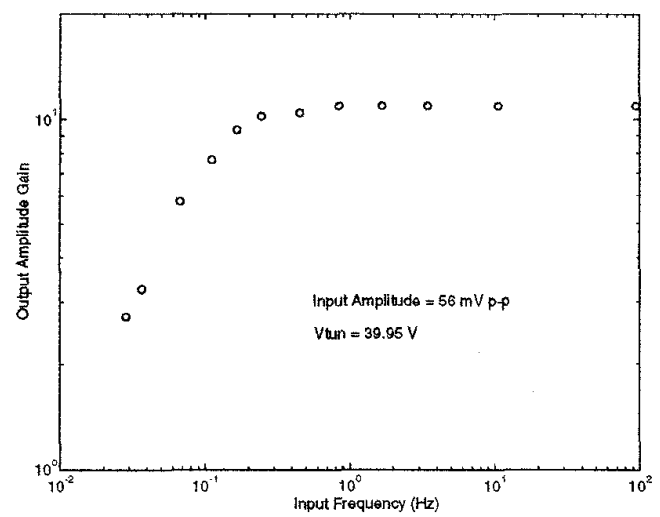

Figure 5. Output Amplitude vs. Frequency for a sine wave input of $56 \mathrm{mV}$ p-p amplitude.

input, preserving the amplified $1 \mathrm{~Hz}$ sine wave. For small signal inputs, we can approximate (7) as

$$
\tau A_{v} \frac{d V_{i n}}{d t}+\tau \frac{d V_{o u t}}{d t}=-\Delta V_{o u t}
$$

where a small signal input is defined as an input that changes the output voltage less than $V_{i n j}$. The resulting transfer function is

$$
V_{o u t}(\omega)=-A_{v} \frac{\omega \tau}{1+j \omega \tau} V_{i n}(\omega)
$$

Figure 5 shows measured output magnitude verses frequency of this amplifier for a $56 \mathrm{mV}$ peak to peak input sine wave.

\section{ACKNOWLEDGEMENTS}

The work was supported by the Office of Naval Research, the Advanced Research Projects Agency, and the Beckman Foundation.

\section{REFERENCES}

11 E.A.Vittoz, "Dynamic Analog Techniques", in Y. Tsividis and P. Antognetti, Design of MOS VLSI Circuits for Telecommunications, Prentice Hall, 1985.

[2] Y. Leblebici and S. M. Kang, Hot Carrier Reliability of MOS VLSI Circuits, Kluwer Academic Publisher, 1993.

[3] B. A. Minch, C. Diorio, P. Hasler, and C. A. Mead, "Translinear Circuits Using Subthreshold FloatingGate MOS Transistors," to appear in Analog Integrated Circuits and Signal Processing, 1996.

[4] P. Hasler, C. Diorio, B. A. Minch, and C. Mead, "Single Transistor Learning Synapses", Advances in Neural Information Processing Systems \%, Morgan Kaufmann Publishers, San Mateo, CA, 1995. 